\title{
DISCRIMINAÇÃO ENTRE ESTÁGIOS NUTRICIONAIS NA CULTURA DO TRIGO COM TÉCNICAS DE VISÃO ARTIFICIAL E MEDIDOR PORTÁTIL DE CLOROFILA ${ }^{1}$
}

\author{
DARLY G. DE SENA JÚNIOR ${ }^{2}$, FRANCISCO DE A. DE C. PINTO 3 , \\ DANIEL M. DE QUEIROZ ${ }^{4}$, NERILSON T. SANTOS ${ }^{5}$, JOSEPH K. KHOURY JÚNIOR
}

RESUMO: O ajuste da adubação nitrogenada é um tema que suscita preocupações econômicas e ambientais em todo o mundo. Isso decorre da elevada resposta das culturas, especialmente gramíneas, ao nitrogênio e da falta de métodos adequados de quantificação de sua disponibilidade no solo. Com o objetivo de avaliar a discriminação de três estágios nutricionais na cultura do trigo, foram utilizadas imagens digitais e um medidor portátil de clorofila (SPAD -502). Os dados foram coletados em três épocas ( $8 ; 14$ e 20 dias após a adubação nitrogenada em cobertura - DAA), em parcelas de trigo submetidas a três doses de $\mathrm{N}\left(0 ; 30\right.$ e $\left.60 \mathrm{~kg} \mathrm{ha}^{-1}\right)$. As imagens foram processadas para desenvolvimento dos classificadores multivariados, utilizando-se de nove índices espectrais com as combinações dos valores médios dos "pixels". Os dados de clorofila e N foliar foram utilizados para desenvolver classificadores univariados. Verificou-se que o sistema de visão artificial foi mais eficiente que o SPAD aos 8 DAA. Aos 14 e 20 DAA, a classificação univariada com os dados SPAD foi equivalente aos classificadores com dados de imagens. Com a utilização das imagens digitais, foi possível discriminar os estágios nutricionais oito dias após a primeira adubação nitrogenada em cobertura.

PALAVRAS-CHAVE: agricultura de precisão, taxas variáveis, processamento de imagens.

\section{MACHINE VISION TECHNIQUES AND MULTIVARIATE CLASSIFIERS FOR NITROGEN FERTILIZATION DOSES DISCRIMINATION IN WHEAT}

\begin{abstract}
Sidedress nitrogen fertilization is currently discussed throughout the world due to its economical and environmental implications. The cereal crops strongly respond to $\mathrm{N}$ application and there is a lack of current methods to determine $\mathrm{N}$ availability on the soil. The aim of this work was to evaluate the discrimination among three nutritional levels in wheat crop using digital images and a portable chlorophyll meter. Data were collected in plots with three levels of $\mathrm{N}\left(0 ; 30\right.$ and $\left.60 \mathrm{kha}^{-1}\right)$ in three dates $(8 ; 14$ and 20 days after sidedress fertilization). The images were processed using nine spectral indices and elaborated multivariate classifiers based on the mean pixel values. The chlorophyll data and leaf nitrogen concentration were used in univariate classifiers. The classification using the machine vision techniques were better than the chlorophyll meter (SPAD) at $8 \mathrm{DAF}$, since the Kappa coefficient was better than a random classification. At 14 and 20 DAF there were no statistical differences between this type of data and the data from images. Using digital images it was possible discriminate the nutritional levels eight days after sidedress fertilization.
\end{abstract}

KEYWORDS: precision agriculture, variable rate fertilization, image processing.

\footnotetext{
${ }^{1}$ Extraído da Tese do primeiro autor. Bolsista do CNPq.

${ }^{2}$ Engo Agrônomo, Prof. Adjunto, Universidade Federal de Goiás, Câmpus Jataí, Fone: (0XX64) 3632.2101, darly.sena@gmail.com

${ }^{3}$ Prof. Adjunto, Ph.D., Dep. Engenharia Agrícola, Universidade Federal de Viçosa, facpinto@ufv.br. Bolsista de Pesquisa do CNPq.

${ }^{4}$ Prof. Adjunto, Ph.D., Dep. Engenharia Agrícola, Universidade Federal de Viçosa, queiroz@ufv.br. Bolsista de Pesquisa do CNPq.

${ }^{5}$ Prof. Adjunto, Ph.D., Dep. Informática, Universidade Federal de Viçosa, nsasntos@dpi.ufv.br

${ }^{6}$ Prof. Adjunto, D.S., Universidade Federal Rural do Rio de Janeiro, khouryjr@ufrrj.br

Recebido pelo Conselho Editorial em: 30-11-2006
}

Aprovado pelo Conselho Editorial em: 7-1-2008 


\section{INTRODUÇÃO}

De modo geral, as culturas agrícolas respondem de forma intensa à adubação nitrogenada, devido a sua baixa disponibilidade no solo e às grandes quantidades requeridas. Esse fato, aliado à ausência de métodos adequados de análise de N no solo (ORTIZ-MONASTERIO, 2002), pode levar ao uso de doses elevadas de N (SCHRÖDER et al., 2000), especialmente em países desenvolvidos. Nessa situação, devido ao baixo custo relativo do fertilizante, é comum a aplicação de doses elevadas pelos produtores com o objetivo de prevenir perdas por deficiência de N. Por outro lado, ORTIZ-MONASTERIO (2002) considera que, nos países em desenvolvimento, a deficiência de nitrogênio é o problema nutricional mais difundido na cultura do trigo. Quando se utilizam doses excessivas de $\mathrm{N}$, além do custo, existe ainda a preocupação ambiental, graças ao potencial poluidor do nitrato, altamente móvel no solo (TUMBO et al., 2002). Deve-se destacar ainda que a fixação industrial do $\mathrm{N}$ para produção de fertilizantes químicos é altamente dependente da energia proveniente de combustíveis fósseis.

A agricultura de precisão pode ser considerada como a habilidade de avaliar e monitorar a atividade agrícola em nível local, com o objetivo de aumentar a eficiência do processo produtivo (QUEIROZ et al., 2000). A otimização no uso dos insumos, além de contribuir para maximizar o retorno financeiro, pode reduzir o impacto ambiental das atividades agrícolas. Um dos pontos fundamentais em agricultura de precisão é a identificação e a mensuração da variabilidade e, para isso, uma das áreas consideradas prioritárias é o desenvolvimento de sensores (STAFFORD, 2000). Especificamente em relação ao nitrogênio, devido à dificuldade de quantificar sua disponibilidade no solo, muitos buscam utilizar plantas como indicadoras. As principais estratégias utilizam características espectrais dos pigmentos foliares, especialmente a clorofila, que apresenta correlação com a disponibilidade de N. Entretanto, outros indicadores têm sido propostos, como os polifenóis (CARTELAT et al., 2005), que podem ser determinados por sua absorbância na faixa do ultravioleta. Esses autores associaram medições de clorofila e polifenóis para acentuar diferenças entre plantas submetidas a diferentes níveis de $\mathrm{N}$.

Um fator fundamental é o estádio de desenvolvimento da cultura, pois se a identificação da deficiência ocorrer em estádio muito avançado, a informação pode não ser útil para a correção da deficiência naquele ciclo de produção (WU et al., 2007). Entretanto, dados coletados com medidores portáteis de clorofila em estádios avançados, próximo ao florescimento, podem ser úteis para a predição de qualidade e produtividade de grãos em trigo (LE BAIL et al., 2005).

A aplicação da reflectância para fins agrícolas teve início nos anos de 1970, quando espectrômetros de infravermelho foram usados para medir a clorofila em folhas de plantas (LUKINA et al., 1997). A reflectância da cultura pode ser medida por radiômetros, que operam em bandas espectrais específicas, ou avaliada por meio de imagens orbitais, aéreas ou obtidas a partir de equipamentos agrícolas (visão artificial). Embora os desafios para a implementação dos sistemas de visão artificial na área agrícola sejam grandes (KASSLER, 2001), os mesmos apresentam grande potencial de utilização na coleta de dados em agricultura de precisão (PINTO et al., 2001). Considera-se que os dados obtidos por sensoriamento remoto são medida direta das condições da cultura, que integra o efeito da maioria dos fatores ambientais (MORIONDO et al., 2007).

Acredita-se que as câmeras digitais se tornarão um dos métodos mais viáveis para a estimativa da refletância do dossel por causa da sua popularização. Além disso, as imagens de alta resolução obtidas com essas câmeras podem permitir a avaliação de outras características como, por exemplo, a textura nas imagens. A principal desvantagem das câmeras digitais é a menor resolução espectral em relação aos radiômetros, mas é uma limitação que pode ser contornada pela utilização de filtros específicos na câmera. Dados coletados com câmeras digitais podem ser utilizados para detectar níveis de estresse nutricional por nitrogênio, em tempo real, para a adubação em cobertura (NOH et al., 2006).

Com o objetivo de minimizar variações causadas por fatores externos e promover o realce dos objetos de interesse, podem ser utilizados índices espectrais (HABOUDANE et al., 2002), que são 
combinações entre bandas espectrais. De acordo com SCHRÖDER et al. (2000), parâmetros obtidos pela combinação da reflectância no vermelho (ou verde) com o infravermelho próximo podem ser bons indicadores da tonalidade de verde da cultura e, conseqüentemente, do teor de clorofila.

No presente trabalho, foi avaliada a hipótese de que é possível discriminar estágios nutricionais na cultura do trigo proporcionados por doses de nitrogênio a partir da estimativa da resposta espectral do dossel por meio de um sistema de visão artificial. O trabalho ainda teve o objetivo de comparar a classificação utilizando imagens digitais e estimativas do teor de clorofila foliar (valores SPAD).

\section{MATERIAL E MÉTODOS}

Os dados foram coletados no Câmpus da Universidade Federal de Viçosa - MG. Foi utilizada a cultivar de trigo BRS 207, com adubação de plantio uniforme, na dose de $300 \mathrm{~kg} \mathrm{ha}^{-1}$ da formulação 8-28-16. Foram implantadas 45 parcelas, com seis linhas espaçadas 0,20 m, comprimento de $5,00 \mathrm{~m}$ e 370 sementes por $\mathrm{m}^{2}$. A adubação em cobertura foi realizada a lanço, 15 dias após a emergência (DAE), utilizando-se de três tratamentos, correspondentes a $0 ; 30$ e $60 \mathrm{~kg}$ de $\mathrm{N} \mathrm{ha}^{-1}$, e 15 repetições. A dose de $60 \mathrm{~kg}_{\text {de }} \mathrm{N} \mathrm{ha}^{-1}$ é a recomendada para cobertura na condição de irrigação, para cultivares sem tendência ao acamamento (SOUZA \& FRONZA, 1999).

Para avaliar os métodos de identificação do estado nutricional das plantas, foram desenvolvidos classificadores estatísticos para discriminar as três classes definidas pelas doses de $\mathrm{N}$ aplicadas em cobertura, utilizando: características extraídas de imagens digitais das plantas e estimativas do teor de clorofila nas folhas (valores SPAD).

As imagens e os valores SPAD foram coletados em três épocas: 23; 29 e 35 DAE, que correspondiam a 8; 14 e 20 dias após a primeira adubação em cobertura (DAA). Imagens coloridas e infravermelhas falsa-cor foram adquiridas simultaneamente, utilizando câmera digital Duncantech MS 3100. De acordo com o fabricante, os sensores da câmera apresentam centro das bandas de espectrais e largura de faixa até o ponto em que metade da transmissão é reduzida a $50 \%$ de 460 45nm; 540 - $40 \mathrm{~nm} ; 670$ - $40 \mathrm{~nm}$ e 800 - $65 \mathrm{~nm}$ para as bandas azul, verde, vermelho e infravermelho, respectivamente.

Foi construído um suporte com altura regulável, no qual a câmera foi fixada. Esse suporte era mantido dois metros acima do solo, enquanto se adquiriam as imagens utilizando um computador e um estabilizador acoplado a uma bateria. Foram obtidas imagens de uma área de aproximadamente $0,70 \mathrm{~m}$ por $0,50 \mathrm{~m}$, o que corresponde à resolução espacial de, aproximadamente, $0,05 \mathrm{~cm}$ "pixel"-1. Em cada parcela, foram obtidas três imagens, em posições diferentes, evitando imagens das bordaduras. Para a obtenção das estimativas do teor de clorofila, utilizou-se de medidor portátil de clorofila SPAD 502, sendo considerado o valor SPAD da parcela a média de 30 valores coletados na folha mais nova, completamente expandida, de plantas ao longo da parcela.

As imagens foram processadas para extração de características (valores digitais) com o sistema computacional Matlab, versão 6.5. Foram utilizadas as bandas verde e vermelha isoladamente, pois trata-se de faixa do espectro eletromagnético, respectivamente, refletida e absorvida pela clorofila. As bandas foram normalizadas com o intuito de reduzir o efeito da variação de luminosidade (GONZALES \& WOODS, 2000). Foram utilizadas três razões simples entre as bandas do vermelho, do verde e do infravermelho com o intuito de acentuar diferenças na refletividade das plantas submetidas às diferentes doses de $\mathrm{N}$ e reduzir o efeito da variação de luminosidade. Foram testados, também, três índices espectrais encontrados na literatura: o NDVI (TUCKER, 1979, citados por WU et al., 2007); GNDVI (GITELSON et al., 1996, citados por WU et al., 2007) e o índice do Excesso de Verde (MEYER et al., 1998). Além desses, testou-se ainda um índice combinando as quatro bandas espectrais, pela modificação do índice do Excesso de Verde, para acentuar a diferença entre as porções refletidas (infravermelho próximo e verde) e absorvidas (vermelho e azul) pela clorofila. 
Sendo assim, de cada posição de aquisição de imagens, foram obtidas nove imagens-índice, criadas pelo processamento com os índices espectrais, de acordo com as eqs. (1), (2), (3), (4), (5), (6), (7), (8) e (9):

$$
\begin{aligned}
& \mathrm{EVd}=\frac{2 \mathrm{Vd}-\mathrm{Vm}-\mathrm{Az}}{\mathrm{Vm}+\mathrm{Vd}+\mathrm{Az}} \\
& \mathrm{RVdVm}=\frac{\mathrm{Vd}}{\mathrm{Vm}} \\
& \mathrm{Vdn}=\frac{\mathrm{Vd}}{\mathrm{Vm}+\mathrm{Vd}+\mathrm{Az}} \\
& \mathrm{Vmn}=\frac{\mathrm{Vm}}{\mathrm{Vm}+\mathrm{Vd}+\mathrm{Az}} \\
& \mathrm{NDVI}=\frac{\mathrm{IV}-\mathrm{Vm}}{\mathrm{IV}+\mathrm{Vm}} \\
& \mathrm{GNDVI}=\frac{\mathrm{IV}-\mathrm{Vd}}{\mathrm{IV}+\mathrm{Vd}} \\
& \mathrm{RVd}=\frac{\mathrm{Vd}}{\mathrm{IV}} \\
& \mathrm{RVm}=\frac{\mathrm{Vm}}{\mathrm{IV}} \\
& \mathrm{IVVd}=\frac{\mathrm{IV}+\mathrm{Vd}-\mathrm{Vm}-\mathrm{Az}}{\mathrm{IV}+\mathrm{Vm}+\mathrm{Vd}+\mathrm{Az}}
\end{aligned}
$$

em que,

EVd - índice do excesso de verde;

Vd - valor do "pixel" na banda do verde;

Vm - valor do "pixel" na banda do vermelho;

Az - valor do "pixel" na banda do azul;

$\mathrm{R}_{\mathrm{VdVm}}$ - razão verde-vermelho;

Vdn - verde normalizado;

Vmn - vermelho normalizado;

NDVI - índice de vegetação por diferença normalizada;

IV - valor do "pixel" na banda do infravermelho próximo;

GNDVI - índice de vegetação por diferença normalizada no verde;

$\mathrm{R}_{\mathrm{Vd}}$ - razão verde-infravermelho;

$\mathrm{R}_{\mathrm{Vm}}$ - razão vermelho-infravermelho, e

IVVd - índice do excesso de infravermelho e verde.

Considerou-se que a média dos valores de nível de cinza dos "pixels" nas imagens-índice foi influenciada pela diferença na absorção e reflexão de energia luminosa pelas plantas submetidas às diferentes doses de nitrogênio, ou seja, relacionada com a refletância do dossel da cultura. Assim, a partir das imagens-índice, foi criado um vetor de dados padronizados para cada uma das 135 posições ( 3 tratamentos x 15 repetições x 3 imagens por parcela). Para reduzir o efeito de escala, as variáveis foram padronizadas de acordo com a eq.(10) (JOHNSON \& WICHERN, 1998).

$$
\mathrm{z}_{\mathrm{p}}=\frac{\mathrm{z}_{\mathrm{i}}-\mathrm{m}}{\sigma}
$$

em que, 
$\mathrm{z}_{\mathrm{p}}$ - valor da variável padronizada;

$z_{i}$ - valor da variável original;

$\mathrm{m}$ - valor médio de $\mathrm{z}$, e

$\sigma$ - desvio-padrão de $z$.

Utilizando-se do sistema computacional Matlab, versão 6.5, foram desenvolvidos classificadores estatísticos para designar cada um dos 135 vetores a uma das três classes definidas pelas doses de $\mathrm{N}$ aplicadas em cobertura. Foram desenvolvidos classificadores utilizando as médias dos valores dos "pixels" nas imagens-índice, para todas as combinações dos nove índices espectrais, o que totalizou 511 combinações, para as três épocas de obtenção das imagens no campo. Assumindo-se que os dados apresentavam distribuição normal e que as probabilidades a priori de ocorrência das classes eram iguais, um vetor desconhecido de valores padronizados (x) foi designado à classe $\mathrm{j}$, cujo valor da função de decisão $\mathrm{d}_{\mathrm{j}}(\mathrm{x})$ foi maior do que em qualquer outra classe $\mathrm{i}$, ou seja, $\mathrm{d}_{\mathrm{j}}(\mathrm{x})>\mathrm{d}_{\mathrm{i}}(\mathrm{x})$ (GONZALES \& WOODS, 1992).

$$
d_{j}(x)=-0,5 \ln \left[\operatorname{det}\left(S_{j}\right)\right]-0,5\left[\left(x-m_{j}\right)^{T} S_{j}^{-1}\left(x-m_{j}\right)\right]
$$

em que,

$\mathrm{x}$ - vetor desconhecido a ser classificado;

$\mathrm{S}_{\mathrm{j}}$ - matriz de co-variância da classe $\mathrm{j}$, e

$\mathrm{m}_{\mathrm{j}}$ - média da classe $\mathrm{j}$.

A fim de avaliar os erros de classificação, utilizou-se da técnica de validação cruzada, deixando uma amostra de fora. O resultado da classificação do estado nutricional por cada combinação de índices foi avaliado elaborando-se a matriz de erros e calculando-se o coeficiente Kappa. A eq.(12) foi utilizada para a estimativa do coeficiente Kappa (HUDSON \& RAMM, 1987).

$$
\hat{\mathrm{K}}=\frac{\mathrm{n} \sum_{\mathrm{i}=1}^{\mathrm{c}} \mathrm{x}_{\mathrm{ii}}-\sum_{\mathrm{i}=1}^{\mathrm{c}} \mathrm{x}_{\mathrm{i} \oplus} \mathrm{x}_{\oplus \mathrm{i}}}{\mathrm{n}^{2}-\sum_{\mathrm{i}=1}^{\mathrm{c}} \mathrm{x}_{\mathrm{i} \oplus} \mathrm{x}_{\oplus \mathrm{i}}}
$$

em que,

$\hat{K}$ - estimativa do coeficiente Kappa;

$x_{i i}$ - valor na linha i e coluna i (diagonal) da matriz de erros;

$x_{i \oplus}$ - total da linha i;

$x_{\oplus i}$ - total da coluna i;

$\mathrm{n}$ - número total de amostras, e

c - número total de classes.

$\mathrm{Na}$ comparação entre os classificadores, utilizou-se do teste $\mathrm{Z}$ para testar a diferença entre dois coeficientes Kappa independentes, a 5\% de significância, utilizando a equação citada por CONGALTON \& MEAD (1983). O coeficiente Kappa obtido por cada classificador também foi comparado por meio do teste $\mathrm{Z}$, a 5\% de significância ,com o valor zero (classificação ao acaso). A variância do coeficiente Kappa foi determinada de acordo com a equação proposta por BISHOP et al. (1975), citados por HUDSON \& RAMM (1987).

Em cada época de obtenção das imagens no campo, foi selecionado o classificador que apresentou o maior coeficiente Kappa entre as 511 combinações dos vetores médios das características extraídas das três imagens de cada parcela. O teste $\mathrm{Z}$, a $5 \%$ de probabilidade, foi utilizado para comparar o coeficiente Kappa do classificador selecionado em cada época, com o coeficiente Kappa obtido, utilizando os valores SPAD na época correspondente. 


\section{RESULTADOS E DISCUSSÃO}

Os resultados dos classificadores do estado nutricional desenvolvidos com valores SPAD e avaliados utilizando-se da validação cruzada são apresentados na Tabela 1.

TABELA 1. Coeficiente Kappa dos classificadores do estado nutricional desenvolvidos com os valores SPAD, em três épocas, após a adubação nitrogenada em cobertura, na cultura do trigo. Kappa coefficient for the classifiers of the nutritional status developed with SPAD values on three epochs after side dress nitrogen fertilization on wheat crop.

\begin{tabular}{cc}
\hline Dias Após a Adubação & Coeficiente Kappa \\
\hline 8 & 0,07 \\
14 & $0,61 * \mathrm{a}$ \\
20 & $0,70 * \mathrm{a}$ \\
\hline
\end{tabular}

* Diferente de zero pelo teste Z, a 5\% de probabilidade.

Os coeficientes Kappa seguidos de mesma letra não diferiram, pelo teste Z, a 5\% de probabilidade.

O coeficiente Kappa do classificador desenvolvido com os valores SPAD coletados, aos 8 DAA, foi estatisticamente igual a zero, pelo teste $\mathrm{Z}$, a $5 \%$ de probabilidade. Isso equivale dizer que essa classificação é estatisticamente igual a uma classificação ao acaso. Com oito dias após a adubação, o teor de clorofila das folhas das plantas de trigo ainda não havia sido influenciado pelas doses de $\mathrm{N}$ aplicadas ou o medidor de clorofila não foi capaz de detectar as diferenças. Por outro lado, aos 14 e aos 20 DAA, as classificações foram $61 \%$ e $70 \%$ melhores que a classificação ao acaso, respectivamente. Os classificadores desenvolvidos aos 14 e 20 DAA não foram estatisticamente diferentes, apesar de MURDOCK et al. (1997) afirmarem que são necessárias três semanas para a resposta ao $\mathrm{N}$ aplicado para utilização do medidor de clorofila SPAD 502 em trigo.

Para os classificadores desenvolvidos com a média dos "pixels" nas imagens-índice, em cada época, considerou-se como o melhor classificador aquele que apresentou o maior valor para o coeficiente Kappa. Os vetores de índices dos melhores classificadores para cada época são apresentados na Tabela 2. Todos os coeficientes Kappa foram estatisticamente diferentes de zero, pelo teste $\mathrm{Z}$, a $5 \%$ de probabilidade.

TABELA 2. Combinações de índices dos melhores classificadores utilizando a média dos valores dos "pixels" nas imagens-índice selecionados pelo coeficiente Kappa, em cada época. Best classifiers indexes combination using mean pixel value on index images selected by Kappa coefficient on each epoch.

\begin{tabular}{clc}
\hline Época & \multicolumn{1}{c}{ Combinação de Índices } & Coeficiente Kappa \\
\hline 8 DAA & GNDVI; IVVd & $0,28^{* a}$ \\
8 DAA & VdVm; NDVI; GNDVI; VmIV & $0,28^{* a}$ \\
14 DAA & Evd; Vdn; VmIV & $0,66^{* b}$ \\
20 DAA & Evd; VdVm; Vmn; GNDVI; VdIV; IVVd & $0,70^{*} \mathrm{~b}$ \\
20 DAA & VdVm; Vdn; ; Vmn; GNDVI; VdIV; IVVd & $0,70 * \mathrm{~b}$ \\
\hline
\end{tabular}

* Diferente de zero, pelo teste $\mathrm{Z}$, a $5 \%$ de probabilidade.

Os coeficientes Kappa seguidos de mesma letra não diferiram, pelo teste Z, a 5\% de probabilidade.

Verifica-se que os coeficientes Kappa dos melhores classificadores na época 14 e 20 DAA diferiram do coeficiente Kappa obtido aos 8 DAA. Dessa forma, tanto nos classificadores desenvolvidos com dados de imagens quanto com valores de SPAD, os coeficientes Kappa apresentaram valores maiores em estádios mais avançados. No caso dos dados extraídos das imagens-índice, todos os coeficientes Kappa diferiram estatisticamente de zero, o que não ocorreu com os valores SPAD. 
O medidor de clorofila utiliza apenas o vermelho e o infravermelho próximo para avaliar o conteúdo de clorofila nas folhas. Entretanto, em todas as combinações selecionadas com os dados das imagens, a banda verde estava presente, indicando sua importância para a classificação. Acredita-se que esse pode ter sido um diferencial que proporcionou melhores resultados ao classificador utilizando os dados das imagens.

Os classificadores desenvolvidos com as características extraídas das imagens mostraram-se superiores estatisticamente ao classificador desenvolvido com os valores SPAD aos 8 DAA. Aos 14 DAA e aos 20 DAA, a diferença entre os coeficientes Kappa não foi significativa (Tabela 3).

TABELA 3. Coeficiente Kappa dos melhores classificadores do estado nutricional com dados de imagens e valores SPAD. Kappa coefficient of the nutritional status best classifiers using image data and SPAD values.

\begin{tabular}{ccc}
\hline Época & Tipo de Classificador & Coeficiente Kappa \\
\hline \multirow{2}{*}{8 DAA } & imagem & $0,28^{*} \mathrm{a}$ \\
& SPAD & $0,07 \mathrm{~b}$ \\
\hline \multirow{2}{*}{$14 \mathrm{DAA}$} & imagem & $0,66^{*} \mathrm{a}$ \\
& SPAD & $0,61^{*} \mathrm{a}$ \\
\hline \multirow{2}{*}{$20 \mathrm{DAA}$} & imagem & $0,70^{*} \mathrm{a}$ \\
& SPAD & $0,70^{*} \mathrm{a}$ \\
\hline
\end{tabular}

* Diferente de zero, pelo teste Z, a 5\% de probabilidade.

Os coeficientes Kappa seguidos de mesma letra em cada época não diferiram, pelo teste $Z$, a $5 \%$ de probabilidade.

Acredita-se que a maior eficiência dos classificadores com as características extraídas das imagens em relação aos classificadores SPAD, aos 8 DAA, deve-se ao tipo de informação extraída. O SPAD realiza estimativa pontual da clorofila nas folhas das plantas, ao passo que a informação extraída das imagens integra informação do dossel da cultura. Assim, ao se utilizar a média dos valores dos "pixels", a informação engloba, além da cor das folhas, informação sobre a cobertura do solo e a variação de tonalidade de verde. Embora a presença do solo nas imagens possa ser prejudicial à classificação, um dos efeitos do $\mathrm{N}$ sobre a cultura é maior vigor com conseqüente maior cobertura do solo. Sendo assim, as parcelas com maior disponibilidade de $\mathrm{N}$ apresentam menor quantidade de solo nas imagens, o que influencia no valor médio dos "pixels". O efeito da presença do solo nas imagens pode ser avaliado removendo-se, por meio de processamento, os "pixels" de solo. SENA JÚNIOR et al. (2007), utilizando esse procedimento, obtiveram coeficientes Kappa iguais com e sem a presença do solo nas imagens. Entretanto, os autores consideraram que, com a presença do solo nas imagens, a classificação foi superior, pelo maior acerto em cada classe.

Uma vez que o nitrogênio é um nutriente bastante móvel na planta, nas plantas deficientes, esse nutriente é mobilizado nas folhas mais velhas e direcionado para as folhas mais novas. Esse é um dos motivos da senescência precoce de folhas em plantas deficientes, o que acarreta nessas plantas gradiente de tonalidade de verde, com as folhas mais velhas mais amareladas. Essa informação não é avaliada pelo medidor de clorofila, mas está presente nas imagens do dossel. Já foi verificado que medições do dossel (índices) se mostraram mais sensíveis à senescência incipiente do que o SPAD, devido às observações pontuais desse no topo do dossel (ADAMSEN et al., 1999). Além disso, trabalhos têm mostrado que, aparentemente, a resposta dos índices de vegetação a variações na disponibilidade de $\mathrm{N}$ está relacionada a alterações no índice de área foliar (WU et al., 2007). Em situações em que a diferença entre os teores de clorofila é pequena, pode ser vantajoso utilizar métodos de extração a medidores de clorofila, pela sua sensibilidade (PINKARD et al., 2006).

Por outro lado, as informações extraídas das imagens estão sujeitas a "ruídos" provocados por fatores ambientais, sendo o principal deles a iluminação. Nesse caso, para minimizar possíveis efeitos da variação de iluminação, além da utilização de índices, poderiam ser amostradas folhas no campo para a obtenção de imagens em condições de iluminação controlada, coletar imagens sob céu nublado ou fotografar junto às plantas uma placa-padrão para posterior normalização das imagens. 
Além da iluminação, a presença de plantas daninhas e/ou falhas no plantio podem prejudicar a classificação.

As imagens podem ser coletadas a partir de equipamentos agrícolas, aeronaves ou até mesmo aeromodelos, o que torna sua aquisição bastante versátil. Por outro lado, o medidor de clorofila utilizado no trabalho exige contato físico com as folhas, o que, muitas vezes, leva à coleta de dados em poucos pontos no campo, o que pode acarretar indicação inadequada da distribuição espacial do estado nutricional da cultura (NOH et al., 2006). Ressalta-se, entretanto, que já se encontram disponíveis no mercado medidores de clorofila capazes de coletar os dados sem necessidade de contato físico.

\section{CONCLUSÕES}

A utilização de imagens digitais para a identificação do estado nutricional de plantas de trigo, aos 14 e aos 20 dias após a adubação, foi equivalente à utilização do medidor portátil de clorofila SPAD 502.

No estádio menos avançado (8 DAA), o classificador, utilizando imagens digitais, foi superior ao classificador envolvendo valores SPAD.

A utilização dos valores SPAD para discriminar entre os três estágios nutricionais da cultura do trigo no campo requereu pelo menos 14 dias após a primeira adubação nitrogenada em cobertura, realizada aos 15 dias após a emergência.

Com a utilização das características extraídas das imagens das parcelas no campo, foi possível discriminar os estágios nutricionais oito dias após a primeira adubação nitrogenada em cobertura.

\section{REFERÊNCIAS}

ADAMSEN, F.J.; PINTER JÚNIOR ,P.J.; BARNES, E.M.; LAMORTE, R.L; WALL, G.W.; LEAVITT, S.W.; KIMBALL, B.A. Measuring wheat senescence with a digital camera. Crop Science, Madison, v.39, n.3, p.719-24, 1999.

CARTELAT, A.; CEROVIC, Z.G.; GOULAS, Y.; MEYER, S.; LELARGE, C.; PRIOUL, J.L.; BARBOTTIN, A.; JEUFFROY, M.H.; GATE, P.; AGATI, G.; MOYA, I.; Optically assessed contents of leaf polyphenolics and chlorophyll as indicators of nitrogen deficiency in wheat (Triticum aestivum L.) Field Crops Research, Amsterdam, v.91, n.1, p.35-49, 2005.

CONGALTON, R.G.; MEAD, R.A. A quantitative method to test for consistency and correctness in photointerpretation. Photogrammetric Engineering \& Remote Sensing, Bethesda, v.49, n.1, p.69-74, 1983.

GONZALES, R.C.; WOODS, R.E. Processamento de imagens digitais. São Paulo: Edgard Blücher, 2000. 509 p.

HABOUDANE, D.; MILLER, J.R.; TREMBLAY, N.; ZARCO-TEJADA, P.J.; DEXTRAZE, L. Integrated narrow-band vegetation indices for prediction of crop chlorophyll content for application to precision agriculture. Remote Sensing of Environment, New York, v.81, n.2-3, p.416-26, 2002.

HUDSON, W.D.; RAMM, C.W. Correct formulation of the Kappa coefficient of agreement. Photogrammetric Engineering \& Remote Sensing, Bethesda, v.53, n.4, p.421-2, 1987.

JOHNSON, R.A.; WICHERN, D.W. Applied multivariate statistical analysis. New Jersey: Prentice Hall, 1998. 816 p.

KASSLER, M. Agricultural automation in the new millennium. Computers and Electronics in Agriculture, Oxford, v.30, n.1-3, p.237-40, 2001.

LE BAIL, M.; JEUFFROY, M.H.; BOUCHARD, C.; BARBOTTIN, A. Is it possible to forecast the grain quality and yield of different varieties of winter wheat from Minolta SPAD meter measurements? European Journal of Agronomy, Amsterdam, v.23, n.4, p.379-91, 2005. 
LUKINA, E.V.; RAUN W.R.; STONE M.L.; SOLIE J.B.; JOHNSON G.V.; LEES H.L.; LARUFFA, J.M.; PHILLIPS S.B. Effect of row spacing, $N$ rate and growth stage on spectral radiance in winter wheat. OSU Soil Fertility Research Highlights. Stillwater: Oklahoma State University, Department of Plant and Soil Sciences, 1997. p.251-8.

MEYER, G.E.; MEHTA, T.; KOCHER, M.F.; MORTENSEN, D.A.; SAMAL, A. Textural imaging and discriminant analysis for distinguishing weeds for spot spraying. Transactions of the ASAE, St. Joseph, v.41, n.4, p.1.189-97, 1998.

MORIONDO, M.; MASELLI, F.; BINDI, M. A simple model of regional wheat yield based on NDVI data. European Journal of Agronomy, Amsterdam, v.26, n.3, p.266-74, 2007.

MURDOCK, L.; JONES, S.; BOWLEY, C.; NEEDHAM, P.; JAMES, J.; HOWE, P. Using a chlorophyll meter to make nitrogen recommendations on wheat. Cooperative Extension Service. Kentucky: University of Kentucky, 1997. 4 p.

NOH, H; ZHANG, Q.; SHIN, B.; HAN, S.; FENG, L. A neural network model of maize crop nitrogen stress assessment for a multi-spectral imaging sensor. Biosystems Engineering, Amsterdam, v.94, n.4, p.477-85, 2006.

ORTIZ-MONASTERIO, J.I. Nitrogen management in irrigated spring wheat. In: CURTIS, B.; RAJARAM, S.; GOMEZ MACPHERSON, H. (Eds.). Bread wheat improvement and production. Rome: FAO, 2002. p.433-52 (Plant Production and Protection Series, 30)

PINKARD, E.A.; PATEL, V.; MOHAMMED, C. Chlorophyll and nitrogen determination for plantation-grown Eucalyptus nitens and E. globulus using a non-destructive meter. Forest Ecology and Management, Amsterdam, v.223, n.1-3, p.211-17, 2006.

PINTO, F.A.C.; SENA JÚNIOR, D.G.; QUEIROZ, D.M.; GOMIDE, R.L. Visão artificial na agricultura de precisão. In: BALASTREIRE, L.A. (Ed.) Avanços na agricultura de precisão no Brasil no período de 1999-2001. Piracicaba: ESALQ/USP, 2001. p.309-16.

QUEIROZ, D.M.; DIAS, G.P.; MANTOVANI, E.C. Agricultura de precisão na produção de grãos. In: BORÉM, A.; GIUDICE, M.P.; QUEIROZ, D.M.; MANTOVANI, E.C.; FERREIRA, L.R.; VALLE, F.X.R.; GOMIDE, R.L. (Eds.). Agricultura de precisão. Viçosa: UFV, 2000. p.1-41.

SCHRÖDER, J.J.; NEETESON, J.J.; OENEMA, O.; STRUIK, P.C. Does the crop or the soil indicate how to save nitrogen in maize production? Reviewing the state of the art. Field Crops Research, Amsterdam, v.66, n.2, p.151-64, 2000.

SENA JÚNIOR, D.G.; PINTO, F.A.C.; QUEIROZ, D.M.; ALVES, E.A.; MAGALHÃES, J.R. Influência do solo na identificação de doses de nitrogênio em trigo utilizando classificadores multivariados com base em imagens digitais. In: SIMPÓSIO BRASILEIRO DE SENSORIAMENTO REMOTO, 13., 2007, Florianópolis. Anais... São José dos Campos: INPE, 2007. p.385-92.

SOUZA, M.A.; FRONZA, V. Trigo. In: RIBEIRO, A.C.; GUIMARÃES, P.T.; ALVAREZ, V.V.H. (Eds). Recomendações para o uso de corretivos e fertilizantes em Minas Gerais, $5^{a}$ aproximação. Viçosa: CFSEMG, 1999. p.328-31.

STAFFORD, J.V. Implementing precision agriculture in the $21^{\text {st }}$ century. Journal of Agricultural Engineering Research, London, v.76, n.3, p.267-75, 2000.

TUMBO, S.D.; WAGNER, D.G.; HEINEMANN, P.H. Hyperspectral-based neural network for predicting chlorophyll status in corn. Transactions of the ASAE, St. Joseph, v.45, n.3, p.825-32, 2002.

WU, J.; WANG, D.; C.J. ROSEN, C.J.; BAUER, M.E. Comparison of petiole nitrate concentrations, SPAD chlorophyll readings, and QuickBird satellite imagery in detecting nitrogen status of potato canopies. Field Crops Research, Amsterdam, v.101, n.1, p.96-103, 2007. 